\title{
SINFONI adaptive optics integral field spectroscopy of the Circinus Galaxy ${ }^{\star}$
}

\author{
F. Mueller Sánchez ${ }^{1}$, R. I. Davies ${ }^{1}$, F. Eisenhauer ${ }^{1}$, L. J. Tacconi ${ }^{1}$, R. Genzel ${ }^{1,2}$, and A. Sternberg ${ }^{3}$ \\ 1 Max-Planck-Institut für extraterrestrische Physik, Giessenbachstrasse, Postfach 1312, 85741 Garching, Germany \\ e-mail: frankmueller@mpe.mpg.de \\ 2 Department of Physics, 366 Le Conte Hall, University of California, Berkeley, CA, 94720-7300, USA \\ 3 School of Physics and Astronomy, Tel Aviv University, Tel Aviv 69978, Israel
}

Received 20 October 2005 / Accepted 12 April 2006

ABSTRACT

\begin{abstract}
Aims. We investigate the star formation activity and the gas and stellar dynamics on scales of a few parsecs in the nucleus of the Circinus Galaxy.

Methods. Using the adaptive optics near infrared integral field spectrometer SINFONI on the VLT, we have obtained observations of the Circinus galaxy on scales of a few parsecs and at a spectral resolution of $70 \mathrm{~km} \mathrm{~s}^{-1} F W H M$. The physical properties of the nucleus are analyzed by means of line and velocity maps extracted from the SINFONI datacube. Starburst models are constrained using the $\mathrm{Br} \gamma$ flux, stellar continuum (as traced via the $\mathrm{CO}$ absorption bandheads longward of $2.3 \mu \mathrm{m}$ ), and radio continuum.

Results. The similarity of the morphologies of the $\mathrm{H}_{2} 1-0 S$ (1) $2.12 \mu \mathrm{m}$ and $\mathrm{Br} \gamma 2.17 \mu \mathrm{m}$ lines to the stellar continuum and also their kinematics, suggest a common origin in star formation. Within $8 \mathrm{pc}$ of the AGN we find there has been a recent starburst in the last $100 \mathrm{Myr}$, which currently accounts for $1.4 \%$ of the galaxy's bolometric luminosity. The similarity of the spatial scales over which the stars and gas exist indicates that this star formation is occuring within the torus; and comparison of the gas column density through the torus to the maximum possible optical depth to the stars implies the torus is a clumpy medium. The coronal lines show asymmetric profiles with a spatially compact narrow component and a spatially extended blue wing. These characteristics are consistent with some of the emission arising in clouds gravitationally bound to the AGN, and some outflowing in cloudlets which have been eroded away from the bound clouds.
\end{abstract}

Key words. galaxies: active - galaxies: individual: Circinus - galaxies: nuclei - galaxies: Seyfert - galaxies: starburst infrared: galaxies

\section{Introduction}

In the context of active galactic nuclei, star formation activity and the gas and stellar dynamics on scales of a few parsecs to a few tens of parsecs can be counted among the main debated issues. The unified model of active galaxies (see Lawrence 1987 for a review) assumes that the inner region of Seyfert 2 galaxies is comprised of a dense circumnuclear torus that hides the nucleus and the broad-line region from our line of sight at nearinfrared and optical wavelengths. The size scales on which models predict the canonical torus vary from an inner edge at $1 \mathrm{pc}$ out to several tens of parsecs (Pier \& Krolik 1992; Nenkova et al. 2002; Schartmann et al. 2005). These crucial size scales are exactly those that can be resolved with SINFONI in the nearest AGN.

The Circinus galaxy, at a distance of $4.2 \pm 0.8 \mathrm{Mpc}$ (Freeman et al. 1977), is an ideal subject to study because of its proximity $\left(1^{\prime \prime}=20 \mathrm{pc}\right)$. It is a large, highly inclined $\left(i=65^{\circ}\right.$ Freeman et al. 1977), spiral galaxy that hosts both a typical Seyfert 2 nucleus and a circumnuclear starburst on scales of 100-200 pc (Maiolino et al. 1998). Evidence for an obscured Seyfert 1 nucleus is provided by the finding of a broad $\left(F W H M=3300 \mathrm{~km} \mathrm{~s}^{-1}\right) \mathrm{H} \alpha$ line component in polarized light (Oliva et al. 1998). Their picture is also supported by recent $\mathrm{X}$-ray observations. Those above

* Based on observations collected at the European Southern Observatory, Chile.
$10 \mathrm{keV}$ suggest direct $\mathrm{X}$-ray detection of the nucleus through a column density of $4 \times 10^{24} \mathrm{~cm}^{-2}$ (Matt et al. 1999). The X-ray spectrum below $10 \mathrm{keV}$ exhibits a flat continuum and a very prominent iron line, indicative of Compton scattering and fluorescent emission from gas illuminated by an obscured X-ray continuum source (Matt et al. 1996). Circinus shows highly ionized gas extending along the minor axis of the galaxy, with a morphology that is reminiscent of the ionization cones seen in other Seyfert galaxies (Marconi et al. 1994a). $\mathrm{H}_{2} \mathrm{O}$ maser emission has been detected, and the masing gas traces a thin accretion disk about $0.4 \mathrm{pc}$ in radius, with, in addition, a fraction of the masers originating outside the disk, in what appears to be an outflow within $\sim 1$ pc of the nucleus and aligned with the ionization cone (Greenhill et al. 2003). Optical and near-infrared spectrophotometry of the nucleus show a typical Seyfert spectrum, including strong coronal lines (Oliva et al. 1994; Prieto et al. 2004). There are also lines from $\mathrm{H}_{2}$ and low-excitation ionic species, both believed to be associated with starforming regions. The distribution and kinematics of the $\mathrm{Br} \gamma$ line have been interpreted in terms of ongoing star formation activity within a few tens of parsecs of the active nucleus (Maiolino et al. 1998). A young stellar population with an age between $4 \times 10^{7}$ and $1.5 \times 10^{8}$ was found between these scales. Recent observations of the Circinus Galaxy in the range between $1-10 \mu \mathrm{m}$ (Prieto et al. 2004), resolve a $K$ s-band source with a $F W H M$ of $\sim 2$ pc and a spectral energy distribution compatible with a dust temperature of $300 \mathrm{~K}$. 
This paper presents high-resolution, nearly diffractionlimited integral field spectroscopic data of the Circinus galaxy in the $K$-band observed with the adaptive optics (AO) assisted imaging spectrograph SINFONI (Bonnet et al. 2004; Eisenhauer et al. 2003b). The physical scale associated with our spatial resolution is $4 \mathrm{pc}$, allowing us to investigate the properties of the nucleus and its interaction with the circumnuclear environment, highlighting the relationship between star formation and galactic nuclear activity.

We describe our observations and the data reduction procedure in Sect. 2. Section 3 describes the features observed in the spectrum of the galaxy and the methods we used to estimate the spatial resolution of our observations. In Sect. 4 we analyze the morphology of the nuclear source and the origin of the observed $K$-band continuum. Section 5 discusses the morphologies of the emission and the absorption, and the age of the starburst at the nucleus. Then, in Sect. 6 we discuss the kinematics of the emission and the mass distribution in the galaxy. Following these results, Sect. 7 deals with the gas density at the nucleus and the physical characteristics of the torus. A study of the detected coronal lines is presented in Sect. 8, and we present our conclusions in Sect. 9.

\section{Observations and data reduction}

The data presented here were obtained on 15 Jul. 2004 during commissioning of SINFONI (Bonnet et al. 2004; Eisenhauer et al. 2003b) on the VLT UT4. The instrument consists of a cryogenic near infrared integral field spectrometer SPIFFI (Eisenhauer et al. 2003a) coupled to a visible curvature adaptive optics (AO) system (Bonnet et al. 2003). SINFONI performs imaging spectroscopy by cutting the two-dimensional field of view into 32 slices and then rearranging each of the slices (slitlets) onto a one-dimensional pseudo longslit, which is dispersed by a grating wheel and at last the spectra are imaged on the detector.

The AO module was able to correct on the nucleus of Circinus (for which it measured $R=14.1 \mathrm{mag}$ ) in seeing of $\sim 0.5^{\prime \prime}$, to reach a resolution of $0.2^{\prime \prime}$ in the $K$-band (see Sect. 4). This performance is good considering that the optical nucleus of Circinus is rather extended rather than point-like. With the appropriate pixel scale selected, the spectrograph was able, in a single shot, to obtain spectra covering the whole of the $K$-band (approximately $1.95-2.45 \mu \mathrm{m}$ ) at a spectral resolution of $R \sim 4200$ for each $0.0125^{\prime \prime} \times 0.025^{\prime \prime}$ pixel in a $0.80^{\prime \prime} \times 0.80^{\prime \prime}$ field of view. A total of 6 sky and 6 on-source exposures of $300 \mathrm{~s}$ each were combined to make the final data cube with a total integration time of $1800 \mathrm{~s}$.

The data were reduced using the SINFONI custom reduction package SPRED (Abuter et al. 2005). This performs all the usual steps needed to reduce near infrared spectra, but with the additional routines for reconstructing the data cube. Following subtraction of the sky frames from the on-source frames, the data were flatfielded and corrected for dead/hot pixels. The data were then interpolated to linear wavelength and spatial scales, after which the slitlets were aligned and stacked up to create a cube. Finally the atmospheric absorption was compensated using the A0V star HD 190285. Flux calibration was performed by comparison to the high spatial resolution broad band $K_{\mathrm{s}}$ image and photometry obtained with NAOS-CONICA of 11.4 mag in a $0.38^{\prime \prime}$ aperture (Prieto et al. 2004), which was also cross-checked with 2MASS photometry.

No additional point-spread function (PSF) calibration frames using stars were taken. This is primarily because, although in principle one can match the brightness of a calibration star on the wavefront sensor to the AGN, it is not possible to replicate either the spatial extent of the AGN or the background galaxy light associated with it - resulting in a potentially considerable mismatch between the science and calibration PSFs (Davies et al. 2004). The spatial resolution has instead been measured using the methods described in Sect. 3.1.

\section{Spectroscopic features and spatial resolution}

The purpose of this section is to present general results extracted from the data to provide an overview of the features observed in the nuclear region of Circinus. A more detailed analysis is given in subsequent sections.

\subsection{Spatial resolution}

A good way to estimate the spatial resolution is by using a broad line in the spectrum, such as a potential observation of Br $\gamma$ emission from the BLR, which at the distance of Circinus is expected to be spatially unresolved. This has the advantage of measuring the spatial resolution directly from the science frames, and includes all effects associated with shifting and combining the cube. Nevertheless, as can be seen in Fig. 1, we have detected only narrow lines, which are spatially resolved in our observations. As a result, and due to the lack of other point sources in the field, we used indirect means to derive our spatial resolution, making use of a NACO image of the galaxy from Prieto et al. (2004) which contains unresolved star clusters over a field of view of $27^{\prime \prime} \times 27^{\prime \prime}$. Two approaches were used to estimate the PSF of our observations by means of the NACO image. One method of estimating the SINFONI PSF effectively consists of deconvolving the SINFONI continuum image with the intrinsic image of the galaxy, since:

$\mathrm{IM}_{\mathrm{S}}=\mathrm{PSF}_{\mathrm{S}} \otimes \mathrm{IM}_{\mathrm{intr}}$

where $\mathrm{IM}_{\mathrm{S}}$ is the Circinus image from SINFONI, PSF $\mathrm{S}$ is the point spread function, and $\mathrm{IM}_{\mathrm{intr}}$ is the intrinsic image of the galaxy. We obtained the intrinsic image in a similar way, by first deconvolving the NACO image with the NACO PSF. In the NACO image, the smooth underlying galaxy profile was approximated by isophotal analysis using the isophote package from IRAF. This was then subtracted, leaving only the compact sources in the field. The narrowest point in this subtracted image was used as the NACO PSF, which was measured directly to have 0.14 " FWHM. The deconvolution of the NACO image with its PSF was performed using the Lucy algorithm (Lucy 1974) also implemented in IRAF. This deconvolved image was rebinned to our pixel scale of $0.0125^{\prime \prime} /$ pix. For the final stage, instead of deconvolving $\mathrm{IM}_{\mathrm{S}}$ with $\mathrm{IM}_{\mathrm{intr}}$, we fit the SINFONI image with the intrinsic image convolved with the parameterized PSF $_{S}$. This avoids the noise amplification which is an inherent feature of deconvolution. The $\mathrm{PSF}_{\mathrm{S}}$ could be well matched by a symmetrical moffat function. Mismatches were minimized by varying the parameters (center, scale, $\alpha$ and $\beta$ ) of a moffat function defined as $I(r)=\left(1+\left(\frac{r}{\alpha}\right)^{2}\right)^{-\beta}$ and then performing a $\chi^{2}$ minimization test, resulting in a FWHM spatial resolution of $0.20^{\prime \prime}$ $(\sim 3.9 \mathrm{pc})$ with $\alpha=0.148^{\prime \prime}$ and $\beta=1.8$ for this method. An elliptical moffat function was also tested as a possible PSF for our observations. However, this did not improve significantly the fit and so was discarded.

In order to avoid the first deconvolution of the method described above, the second approach to derive the SINFONI PSF 


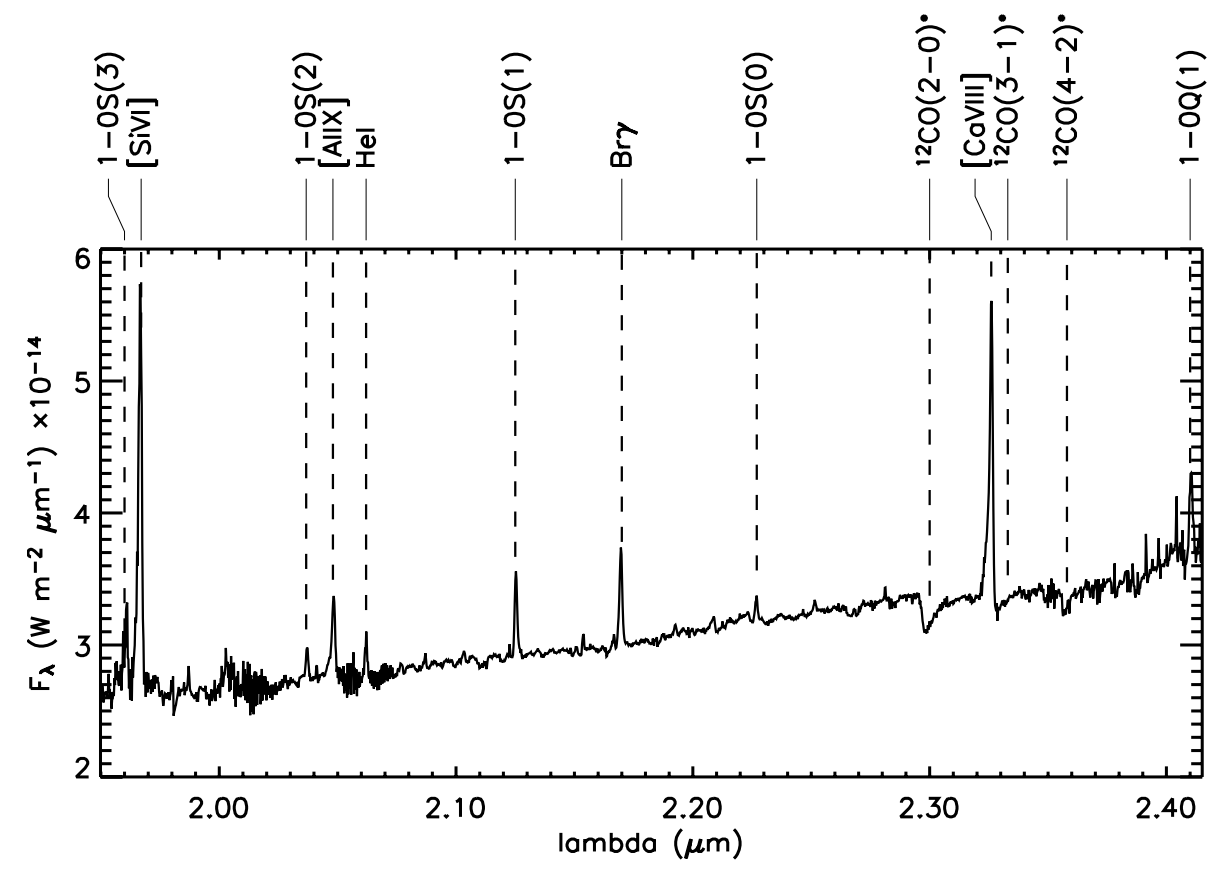

Fig. 1. Nuclear spectrum of Circinus, extracted from the SINFONI datacube in a $0.8^{\prime \prime}$ square aperture. Labels marked with an asterisk represent stellar CO absorption bandheads. The effect of dilution on these bandheads is clearly seen. consists of convolving the NACO PSF with a parameterized degradation function, the latter being obtained by fitting the SINFONI image with the NACO image convolved with the same function. A symmetrical moffat function was used as degradation function. Once again, mismatches were minimized by varying the parameters of the moffat function and then performing a $\chi^{2}$ minimization. The SINFONI PSF was obtained by convolving the NACO PSF with the degradation function. The final $F W H M$ resolution estimated in this way was $0.22^{\prime \prime}(\sim 4.2 \mathrm{pc})$ with $\alpha=0.219^{\prime \prime}$ and $\beta=3.2$.

The confidence on the resulting PSF for both cases was evaluated by the residual of the fitting the following function which considers the difference between the data and model, weighted according the flux in each pixel, and normalised by the total flux in the frame:

Residual $=\frac{\sqrt{\sum\left(\left(\mathrm{IM}_{\mathrm{S}}-\mathrm{Model}^{2} \cdot \mathrm{IM}_{\mathrm{S}}\right)\right.}}{\left(\sum\left(\mathrm{IM}_{\mathrm{S}}\right)\right)^{1.5}} \cdot 100$

where $\mathrm{IM}_{\mathrm{S}}$ is the SINFONI continuum image as above and Model is our constructed image - either the intrinsic galaxy profile convolved with the SINFONI PSF, or the NACO image convolved with the degradation function. The numerator is the quantity minimized during the fit, which was then normalized using the integrated image of the continuum. The residual of the fit in both cases was evaluated as $0.1 \%$, indicating that both methods performed equally well in estimating the PSF. We chose to adopt the PSF obtained by the second approach because no deconvolution is performed.

\subsection{Distribution of emission and absorption features}

In Fig. 1 we present a spectrum of Circinus at a spectral resolution of $R \sim 4200$, integrated over a $0.8^{\prime \prime}$ square aperture centered on the nucleus. The most prominent of the emission lines are the coronal lines [SivI] at $1.96 \mu \mathrm{m}$ and the [CaVIII] at $2.32 \mu \mathrm{m}$. We also detect the [AlIX] $2.04 \mu \mathrm{m}$ line, first mentioned by Maiolino et al. (1998). In addition to these high excitation lines, the $\mathrm{H}_{2} 1-0 S$ (1) $2.12 \mu \mathrm{m}$ and $\mathrm{Br} \gamma 2.17 \mu \mathrm{m}$ emission lines are clearly recognizable. The line fluxes, which are presented
Table 1. Measured nuclear emission line fluxes for Circinus. All measurements are given for a $0.8^{\prime \prime}$ circular aperture centered on the continuum peak.

\begin{tabular}{lcc}
\hline \hline Line & $\begin{array}{c}\lambda^{a} \\
(\mu \mathrm{m})\end{array}$ & $\begin{array}{c}\text { Flux }^{b} \\
\left(10^{-18} \mathrm{Wm}^{-2}\right)\end{array}$ \\
\hline $\mathrm{H}_{2} 1-0 S(3)$ & 1.9576 & 6.0 \\
{$[\mathrm{SiVI}]$} & 1.9634 & 53.9 \\
$\mathrm{H}_{2} 1-0 S(2)$ & 2.0338 & 3.8 \\
{$[\mathrm{AlIX}]$} & 2.040 & 10.0 \\
{$[\mathrm{HeI}]$} & 2.0587 & 5.0 \\
$\mathrm{H}_{2} 1-0 S(1)$ & 2.1218 & 9.0 \\
$\mathrm{Br} \gamma$ & 2.1661 & 14.1 \\
$\mathrm{H}_{2} 1-0 S(0)$ & 2.2233 & 3.4 \\
{$[\mathrm{CaVIII}]$} & 2.3213 & 36.9 \\
$\mathrm{H}_{2} 1-0 Q(1)$ & 2.4066 & 3.4 \\
\hline
\end{tabular}

${ }^{a}$ Wavelengths are in the rest frame.

${ }^{b}$ Uncertainities are approximately $3.2 \times 10^{-20} \mathrm{~W} \mathrm{~m}^{-2}$.

in Table 1, are comparable to previous measurements (Maiolino et al. 1998).

The $K$-band flux density, calculated from the continuum image shown in Fig. 2 within an aperture of $0.8^{\prime \prime}$ is $3.1 \times$ $10^{-14} \mathrm{~W} \mathrm{~m}^{-2} \mu \mathrm{m}^{-1}$ (approximately $50 \mathrm{mJy}$ ). The stellar features, traced by the $\mathrm{CO}$ bandheads, are also distinguishable in the spectrum. However, not all of the stellar features are diagnostically useful. For example, the ${ }^{12} \mathrm{CO}(3-1)$ band is partially filled by the [CaVIII $]$ emission line, and the rest of the bandheads with wavelengths longer than $2.34 \mu \mathrm{m}$ are affected by residual atmospheric features or other emission lines. Therefore, only the ${ }^{12} \mathrm{CO}(2-0)$ band was useful to extract information about the fraction of the nuclear flux that is indeed stellar.

Images of the $2.2-\mu \mathrm{m}$ continuum and the $\mathrm{Br} \gamma, \mathrm{H}_{2}(1-0) S(1)$, [Ca VIII], and [Si VI] line emission, as well as the ${ }^{12} \mathrm{CO}(2-0)$ bandhead flux are presented in Fig. 2. The location of the nucleus, as defined by the centroid of the continuum emission, has been marked with an encircled cross in all maps. This source is offset by $\sim 0.15^{\prime \prime}$ to the south-east of the peak of the $\operatorname{Br} \gamma$ and $\mathrm{H}_{2}$ 1-0 S (1) line emission as well as of the stellar light. Such an offset is consistent with the results of Prieto et al. (2004) 

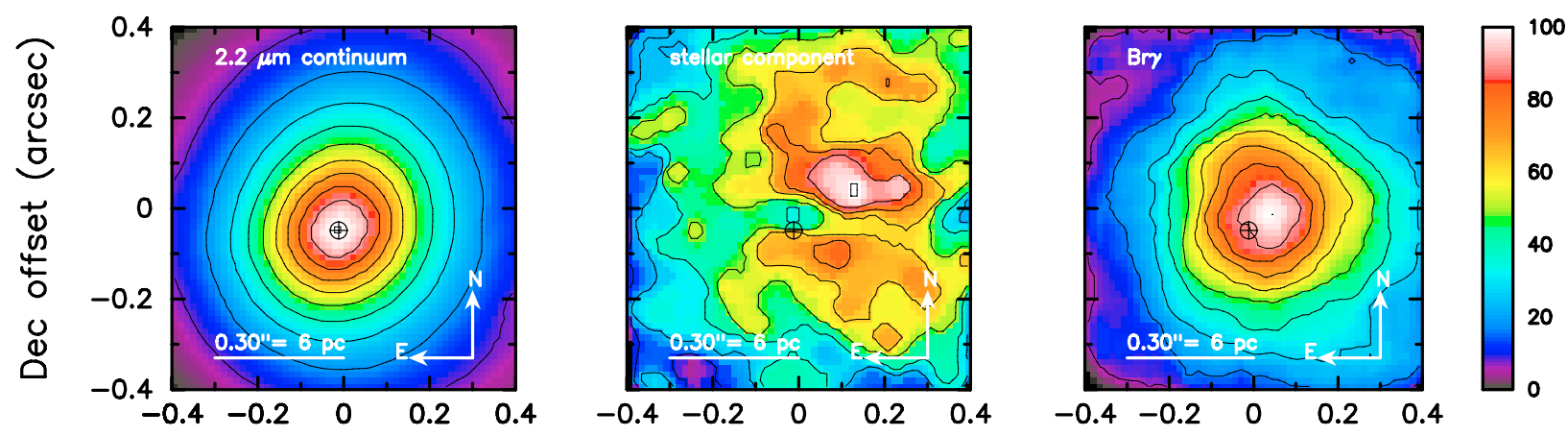

\section{R.A. offset (arcsec)}
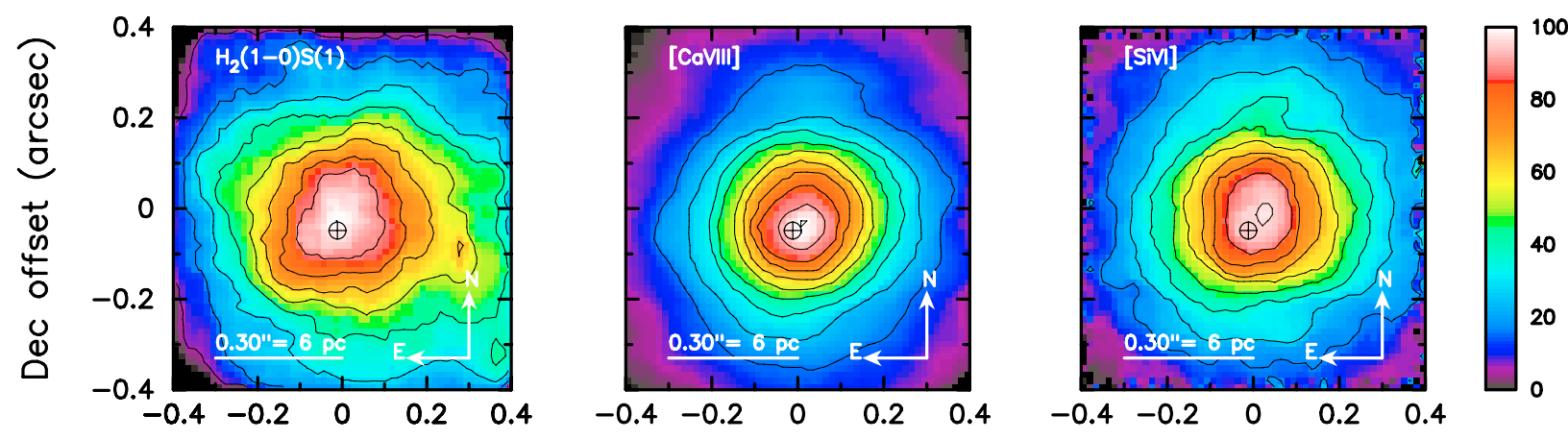

R.A. offset (arcsec)

Fig. 2. Intensity images extracted from the SINFONI data cube in the central arcsec of Circinus. In each case, the colour scale extends from $0-100 \%$ of the peak flux, and contours are spaced equally between $20 \%$ and $90 \%$ of the peak flux. An encircled cross indicates in each case the peak of the continuum emission. The maps show, starting from the left corner, top left: $2.2 \mu \mathrm{m}$ continuum, top center: stellar component (flux contained in the stellar absorption bandhead $\left.{ }^{12} \mathrm{CO}(2-0)\right)$, top right: $\mathrm{Br} \gamma$, bottom left: $\mathrm{H}_{2} 1-0 \mathrm{~S}(1)$, bottom center: [Ca VIII], and bottom right: [Si VI].

who found that the center of the $K$-band radiation is located at $\sim 0.15^{\prime \prime}$ south-east of the brightest central emission seen in the $J$-band and HST F814W images. In contrast to these authors who argued that the $K$-band source is fully obscured at shorter wavelengths and that the emission at these shorter wavelengths is due to nuclear light scattered by the compact dusty structure surrounding the Circinus nucleus, our results suggest a different interpretation because the offset remains even in the $K$-band but only for particular components of the line and continuum emission. We argue that, at least for near infrared emission, the offset arises because the star formation is centered $\sim 0.15^{\prime \prime}$ to the north west of the non-stellar continuum peak. The issue of extinction is addressed further in Sect. 7.

\section{Nuclear dust emission}

The observed equivalent width of the ${ }^{12} \mathrm{CO}(2-0)$ bandhead was used to obtain the fraction of the nuclear flux from the stars in this region, by assuming that the intrinsic (i.e. purely stellar) equivalent width in the nuclear region must be consistent with values predicted by the models created with the stellar population synthesis code STARS (Sternberg 1998; Thornley et al. 2000; Davies et al. 2003). We used the measured flux of the ${ }^{12} \mathrm{CO}(2-0)$ band to estimate the amount of non-stellar continuum using the equation:

$1-D=\frac{E W_{\text {obs }}}{E W_{\text {intr }}}$ where $D$ is the dilution factor, that is the ratio of stellar to total continuum, and $E W_{\text {obs }}$ and $E W_{\text {intr }}$ are the observed and intrinsic stellar equivalent widths respectively. The intrinsic equivalent width is nearly independent of star formation history for any ensemble of stars as predicted by the STARS models, having an almost constant value of $\sim 12 \AA$ assuming solar metallicity (Davies et al. 2006). The observed stellar equivalent width in the central $0.8^{\prime \prime}$ was found to be $\sim 1.8 \AA$, implying that $\sim 85 \%$ of the total nuclear $K$-band luminosity in this region originates from the non-stellar component, a fraction that increases on smaller scales. In Fig. 3, the $K$-band continuum azimuthally averaged radial surface brightness profile is plotted along with those of the PSF and stellar and non-stellar components. The stellar component represents the radial profile of the stellar absorption bandhead ${ }^{12} \mathrm{CO}(2-0)$ image in Fig. 2. All profiles are normalized to their respective peak values; and the stellar profile is centered $0.15^{\prime \prime}$ north west of the non-stellar profile. The predominance of the non-stellar component on these scales is vividly demonstrated by the similarity of the non-stellar and total $K$-band profiles, as well as by the red spectral slope in Fig. 1.

Such nuclear $K$-band emission is interpreted as thermal radiation from hot dust which is heated by the intense UV and $\mathrm{X}$-ray radiation emitted by the Seyfert nucleus. It is often assumed that the dust is close to the sublimation limit $(\sim 1600 \mathrm{~K})$ since it is the hottest emission which will have the greatest impact in the near infrared. However, the slope of the nuclear spectrum in the $K$-band indicates a temperature (unreddened) closer to $740 \mathrm{~K}$, similar to that found for NGC 1068 by 


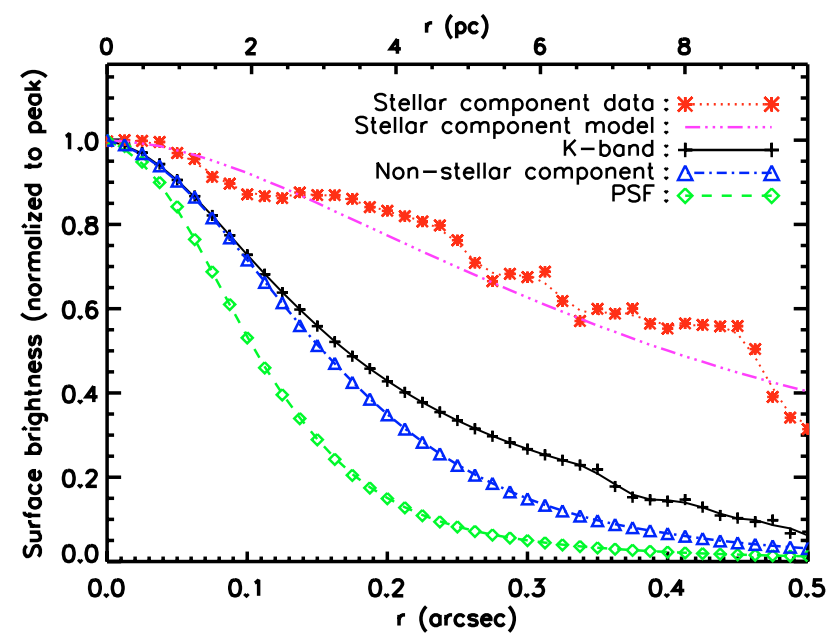

Fig. 3. Radial profile of the nuclear $K$-band surface brightness obtained from an integrated image of the SINFONI datacube (crosses connected by a solid line). The SINFONI $K$-band stellar component (same as stellar continuum) is represented by asterisks connected by a dotted line. The triple-dotted-dashed line represents a model fitted to the stellar component data, which is the exponential profile convolved with the PSF. Open triangles connected by a dotted-dashed line indicate the fraction of the $K$-band radiation which does not come from stars. The open diamonds connected by a short-dashed line indicate the PSF profile.

Thatte et al. (1997), but significantly more than the $300 \mathrm{~K}$ derived by Prieto et al. (2004) from modelling the broad band colours. Since the spectral range of the $K$-band is smaller than the $1-10 \mu \mathrm{m}$ interval used by Prieto et al. (2004), the spectral slopes in these ranges are different, but nevertheless compatible. The estimated temperature of $\sim 700 \mathrm{~K}$ from the $K$-band continuum characterizes only the hottest dust, while $300 \mathrm{~K}$ dust would dominate the mid-infrared SED.

As can be seen from Fig. 3, the non-stellar $K$-band source is marginally resolved. Indeed, a quadrature correction of its FWHM $\left(\sim 0.3^{\prime \prime}\right)$ with that of the PSF yields an intrinsic size of $\sim 0.2^{\prime \prime}(3.9 \mathrm{pc})$. The factor of 2 discrepancy with the size reported by Prieto et al. (2004) arises primarily from the spatial resolution estimated in the NACO data. Compared to their $0.16^{\prime \prime}$ our estimate is $0.14^{\prime \prime}$, which would give a source size of $\sim 0.15^{\prime \prime}$. This emphasises the difficulty of estimating the intrinsic size of only marginally resolved sources. Within the uncertainty of about $0.05^{\prime \prime}$, the sizes are consistent with each other and with those predicted by the torus models (Pier \& Krolik 1992, 1993; Nenkova et al. 2002) in which the inner edge of the dust torus lies $\sim 1$ pc away from the AGN.

\section{Nuclear star formation}

Images of the $\mathrm{Br} \gamma$ and $\mathrm{H}_{2}$ 1-0 S(1) line emission, which are often associated with star formation, are presented in Fig. 2. As the AGN is highly obscured (see Oliva et al. 1998 and Matt et al. 1996, 1999), no broad line region is visible. Several lines of evidence suggest that the $\mathrm{Br} \gamma$ emission is associated with star formation activity surrounding the Seyfert nucleus rather than the narrow line region and ionisation cone. These are the similarity of the morphologies of the $\mathrm{Br} \gamma,{ }^{12} \mathrm{CO}(2-0)$, and $\mathrm{H}_{2}$ 1-0 S(1) emission: the ${ }^{12} \mathrm{CO}(2-0)$ is only slightly more extended than the two lines, and all three are offset to the north west. Additional support is provided by the consistency and uniformity of the $\mathrm{Br} \gamma$ and $\mathrm{H}_{2} 1-0 S$ (1) velocity fields and dispersion maps (see Sect. 6 and Fig. 7). In particular, the velocity field of the Br $\gamma$ has a

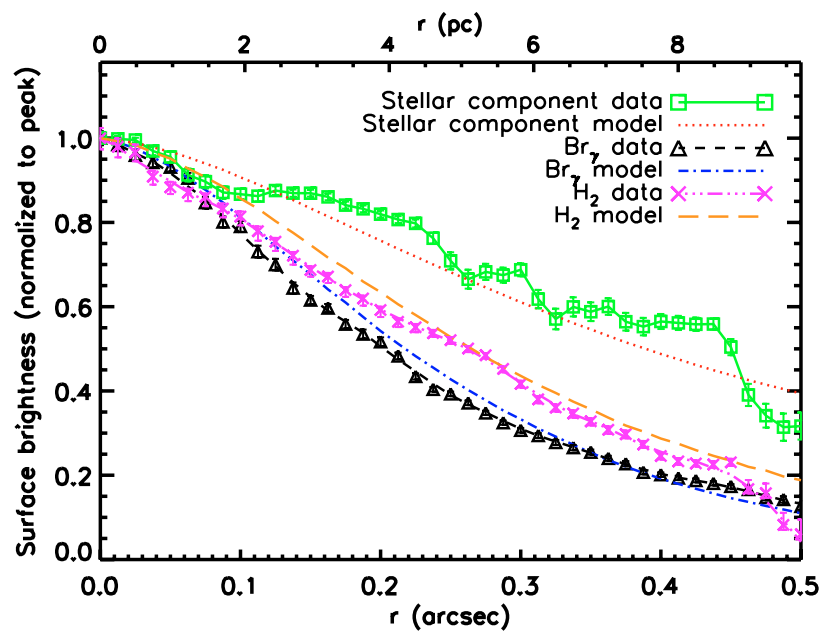

Fig. 4. Radial profile of the surface brightness of the $\mathrm{H}_{2} 1-0 \mathrm{~S}$ (1) (crosses connected by a triple-dotted-dashed line) emission line. The long-dashed line represents a model fitted to the linemap, which is the exponential profile convolved with the PSF. The narrow $\mathrm{Br} \gamma$ radial profile is represented by open triangles connected by a short-dashed line and its model by the dotted-dashed line. Open squares connected by a solid line indicate the nuclear stellar profile, whereas its model is represented by the dotted line.

gradient matching that of the galaxy's major axis rather than showing signs of outflow along the minor axis. We note in fact that the $\mathrm{H}_{2} 1-0 S$ (1) appears to be slightly more extended than the $\mathrm{Br} \gamma$, particularly at weaker levels westwards in the direction of the ionization cone (Maiolino et al. 1998); and it is only in this region that there are significant differences between their velocity fields, with the $1-0 S$ (1) showing signs of outflow. Figure 4 shows the azimuthally averaged surface brightness radial profiles of the $\mathrm{H}_{2} 1-0 S(1)$ and narrow $\mathrm{Br} \gamma$ emission lines and the ${ }^{12} \mathrm{CO}(2-0)$ stellar absorption bandhead.

Both an exponential profile and $r^{1 / 4}$ de Vaucouleurs profile provide good matches to each of these. We prefer to parameterise the profile with the former, because of the clear evidence of a circumnuclear disk from the existence of certain features (namely a bar, Maiolino et al. 2000; and a ring, Marconi et al. 1994b). At these scales, less than $20 \mathrm{pc}$, the $\mathrm{Br} \gamma$, and $\mathrm{H}_{2} 1-0 S$ (1) data are characterised by a disk-scale length $r_{\mathrm{d}}=4_{-0.2}^{+0.1} \mathrm{pc}$. Due to the symmetry in the morphology of the $\mathrm{Br} \gamma$, and $\mathrm{H}_{2} 1-0 S$ (1) profiles, the profiles were not corrected for inclination. This would perhaps be a surprising result for an inclined disk, but indeed the kinematics and simulations suggest that the thickness of the disk plays an important part in the observed morphology: a disk inclined at $65^{\circ}$ with a thickness of only 4 pc $F W H M$ - equivalent to an exponential scale height of $1.7 \mathrm{pc}-$ is consistent with the symmetrical morphology. We return to this point in Sect. 6 where we discuss the kinematics in more detail.

The complex morphology of the stellar component was analyzed in more detail by several simulations. Since the observed stellar luminosity is expected to be produced by several clusters, simulations were carried out in order to study the way the clusters superpose to create the observed complex morphology. In the simulations each cluster was defined by two parameters: its position inside the field, and its mass. The positions were assigned randomly following the radial distribution of the stellar component in Fig. 4, so that the probability of finding a cluster in any position depends on an exponential profile slightly more extended than the line emission. For the mass distribution, systems of young clusters, including super star clusters which are 

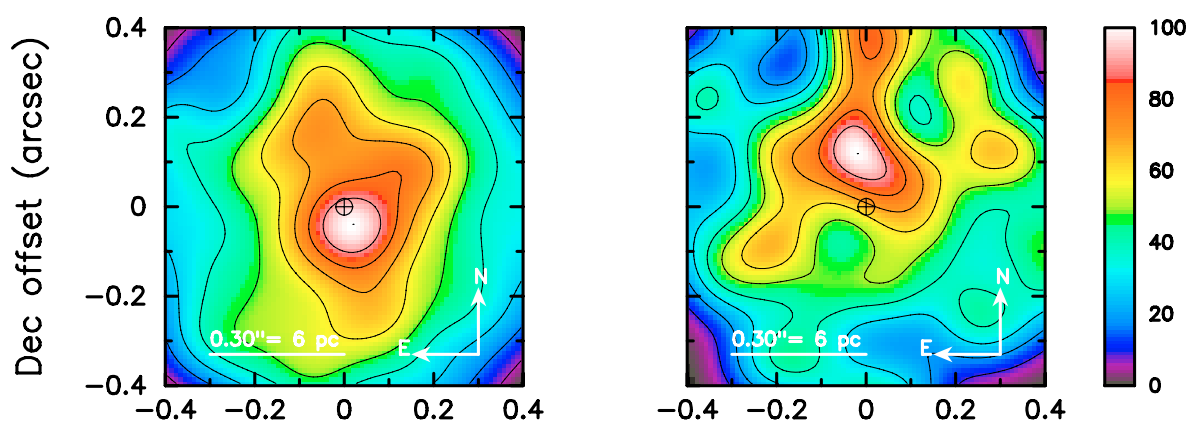

R.A. offset (arcsec)

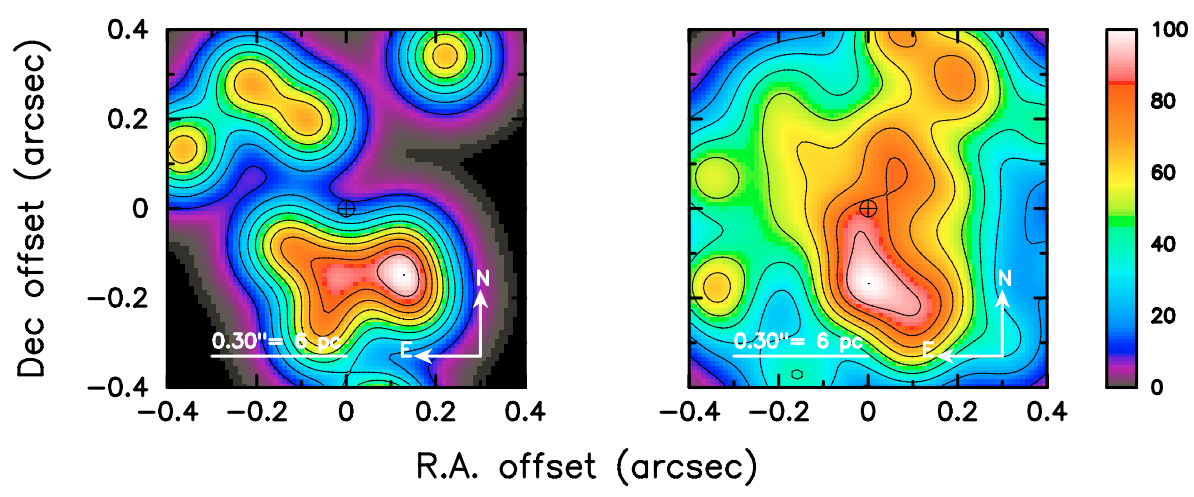

Fig. 5. Intensity images of the star clusters simulations in the central arcsec of Circinus. In each case, the colour scale extends from $0-100 \%$ of the peak flux, and contours are spaced equally between $10 \%$ and $90 \%$ of the peak flux. An encircled cross indicates in each case the center of the region. The maps show, starting from the left corner, top left: 1000 star clusters with constant mass $1 \times 10^{3} M_{\odot}$, top right: 100 star clusters with constant mass $1 \times 10^{4} M_{\odot}$, bottom left: 10 star clusters with constant mass $1 \times 10^{5} M_{\odot}$, and bottom right: 300 star clusters with masses varying from $1 \times$ $10^{3} M_{\odot}$ to $5 \times 10^{4} M_{\odot}$.

preferentially found at the very heart of starbursts, appear to be well represented by a power law mass and luminosity function with $\alpha=-2$. The range of masses was from $1 \times 10^{3}$ to $3 \times 10^{5} M_{\odot}$ which are typical values for star clusters (Meurer et al. 1995). Each cluster was characterized by a two dimensional Gaussian function with $F W H M=0.2^{\prime \prime}$, our spatial resolution. The clusters were generated randomly over a circular field of $0.5^{\prime \prime}$ radius. According to the mass distribution, a mass was assigned to each of them until the observed stellar mass in the region was reached, namely $1 \times 10^{6} M_{\odot}$ (see below). Each cluster had the same mass-to-light ratio. The summation of all clusters over the whole field yields the overall morphology of the stellar component. Figure 5 shows the results of the simulations. It can be seen from Fig. 5 that when massive star clusters are present in the field, the clumpiness of the whole set increases. On the other hand, for a low mass distribution the field remains uniform and symmetric, and therefore the clumpiness decreases.

The observed morphology in Fig. 2 can be best reproduced by a set of star clusters with a mass distribution ranging from $1 \times 10^{3}$ to $5 \times 10^{4} M_{\odot}$, or alternatively a constant mass with value of $1 \times 10^{4} M_{\odot}$, although other scenarios cannot be excluded . Our simulations indicate that the observed complexity and asymmetry of the morphology of the stellar component could easily arise from the superposition of a number of star clusters with typical mass $10^{4} M_{\odot}$.

We determine the age of the star formation by means of two diagnostics: the intrinsic equivalent width of the $\mathrm{Br} \gamma$ line and the supernova rate to stellar $K$-band luminosity ratio $\left(v_{\mathrm{SN}} / L_{K}^{*}\right)$ in the nuclear region. These provide independent information on the star formation history, the former representing the number of hot young blue supergiant stars and the latter the number of supernova remnants, both normalised to the number of later type red giant and supergiant stars. By comparing the two diagnostics to models, the age of the starburst can be satisfactorily constrained. The $E W(\mathrm{Br} \gamma)$ was estimated from the observed
$\mathrm{Br} \gamma$ flux and the stellar continuum (i.e. corrected for dilution by subtracting the continuum contribution from hot dust), resulting in $E W(\mathrm{Br} \gamma)=30 \AA$ in a $0.8^{\prime \prime}$ aperture. The supernova rate $v_{\mathrm{SN}}$ was obtained using the empirical relation presented in Condon (1992) with a spectral index $\alpha=0.1$ (as measured between 5 and $8.64 \mathrm{GHz}$ ), and a flux density at $8.64 \mathrm{GHz}$ of $S_{10}=12 \mathrm{mJy}$, resulting in a value of $v_{\mathrm{SN}}=2.21 \times 10^{-4} \mathrm{yr}^{-1}$. The radio flux density was calculated by means of the $1^{\prime \prime}$ resolution radio image from Davies et al. (1998). The flux density at $8.64 \mathrm{GHz}$ was summed over an aperture of $0.8^{\prime \prime}$, to be consistent with our fluxes, giving $14 \mathrm{mJy}$. Then the thermal contribution was calculated as given in Condon (1992) for a standard electron temperature of $1 \times 10^{4} \mathrm{~K}$. The resulting $2 \mathrm{mJy}$ was subtracted from the total flux density at $8.64 \mathrm{GHz}$. Dividing $v_{\mathrm{SN}}$ found in this way by the stellar $K$-band luminosity $L_{K}^{*}$ of $1.5 \times 10^{6} L_{\odot}$ found after multiplying the observed $K$-band luminosity $L_{K}$ by the dilution factor $D$, yielded a ratio of $1.5 \times 10^{-10} L_{\odot}^{-1} \mathrm{yr}^{-1}$.

We have used the evolutionary synthesis code STARS (Sternberg 1998; Thornley et al. 2000; Davies et al. 2003) to determine the star formation history assuming an exponentially decaying star formation rate of the form $\exp \left(-t / t_{\mathrm{scl}}\right)$, where $t_{\mathrm{scl}}$ is the burst decay time scale and $t$ is the age of the star cluster. We make the standard assumptions of solar metallicity, and a Salpeter IMF of slope $\alpha=-2.35$ in the range $1-120 M_{\odot}$. An important advantage of this model is that it allows us to study not only instantaneous and continuous scenarios, but also finite star formation timescales.

We obtain different ages for the starburst depending on the timescale of the star formation. We consider 4 timescales: continuous star formation with $t_{\mathrm{scl}}=10^{9} \mathrm{yr}$, an instantaneous burst with $t_{\mathrm{scl}}=10^{6} \mathrm{yr}$, and two intermediate scenarios with $t_{\mathrm{scl}}=10^{7}$ and $10^{8}$ yr. First, we compared the calculated intrinsic Br $\gamma$ equivalent width with that from STARS. Figure 6 shows the evolution of $E W(\mathrm{Br} \gamma), v_{\mathrm{SN}} / L_{K}^{*}, M_{*} / L_{K}^{*}$ and $L_{\mathrm{bol}}^{*} / L_{K}^{*}$ as functions of cluster 

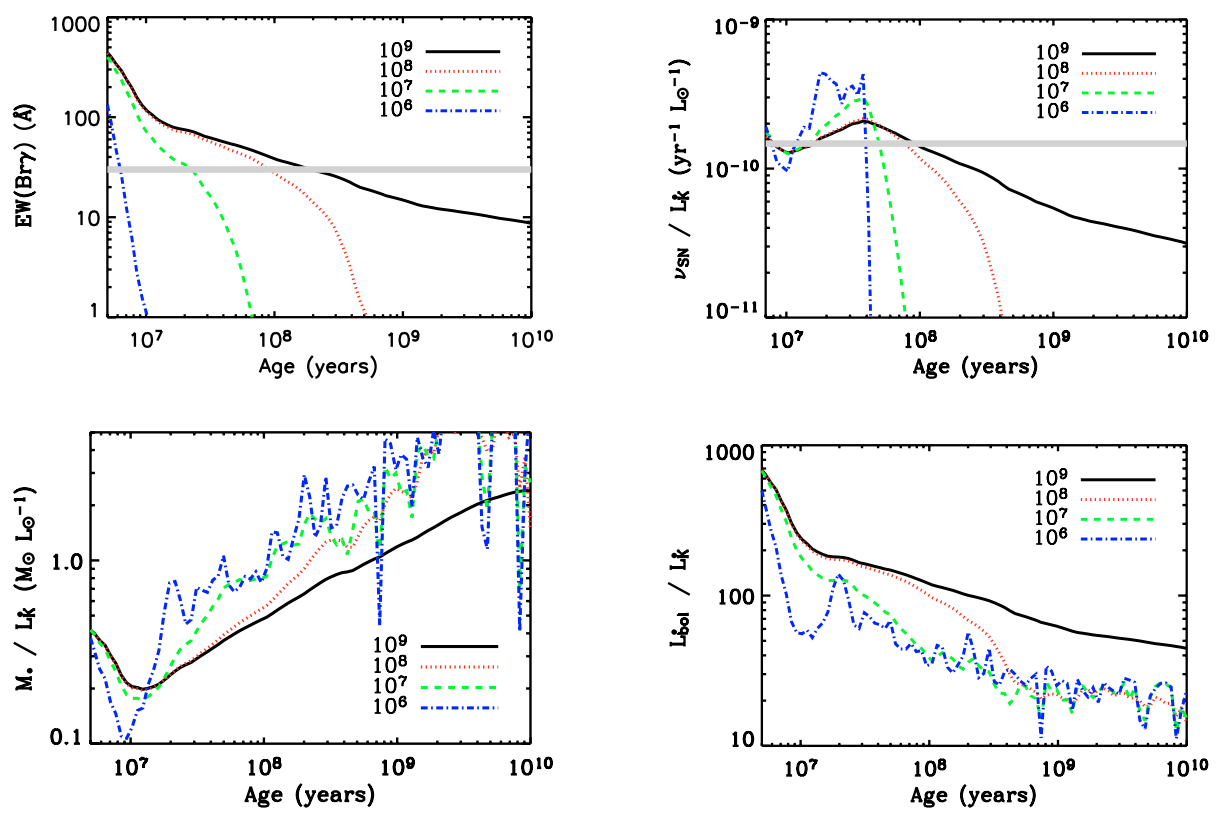

Fig. 6. Evolution of $E W(\mathrm{Br} \gamma)$ (top left), $v_{\mathrm{SN}} / L_{K}^{*}$ (top right), $M_{*} / L_{K}^{*}$ (bottom left) and $L_{\mathrm{bol}}^{*} / L_{K}^{*}$ (bottom right) with age for a stellar cluster with four star formation histories. The gray bands indicate the range of the observed ratios. An asterisk indicates that the parameter refers to its stellar component.

age for each model. It can be seen from this figure that $E W(\mathrm{Br} \gamma)$ alone cannot constrain the age since all scenarios are plausible.

Using the ratio $v_{\mathrm{SN}} / L_{K}^{*}$ together with $E W(\mathrm{Br} \gamma)$, we note that within the central $R<8 \mathrm{pc}$, the constant and the instantaneous star formation models do not fit completely both of the observational constraints $\left(E W(\mathrm{Br} \gamma)=30 \AA\right.$ and $v_{\mathrm{SN}} / L_{K}^{*}=$ $\left.1.47 \times 10^{-10} L_{\odot}^{-1} \mathrm{yr}^{-1}\right)$.

The star formation timescales $t_{\mathrm{scl}}=10^{7}$ and $t_{\mathrm{scl}}=10^{8} \mathrm{yr}$ do fit these constrains from the intersections points at $2 \times 10^{7}$ and $8 \times 10^{7} \mathrm{yr}$ respectively. While the data are consistent with these two scenarios, the shorter timescale would require a degree of fine-tuning, particularly since the supernovae responsible for the radio conntinuum are only just beginning to appear and $E W(\mathrm{Br} \gamma)$ is decaying relatively quickly. Thus there is only a small range of possible ages for this timescale. At an age of $80 \mathrm{Myr}$, the constraints give rise to a wider range of allowed ages, suggesting this is the more likely scenario. Crucially, in either case the data indicate the presence of a young stellar population within a few parsecs of the active nucleus which is now less than half as intense as when it began.

For the preferred star formation scenario given above we find a current mass to $K$-band luminosity ratio of $\sim 0.6 M_{\odot} / L_{\odot}$, yielding a stellar mass of $M_{*} \approx 1 \times 10^{6} M_{\odot}$. Another important parameter to analyze is the bolometric luminosity attributable to the nuclear star formation. Figure 6 shows the evolution of the bolometric luminosity to $K$-band luminosity ratio for an ensemble of stars. From this Figure we obtain $L_{\text {bol }}^{*} / L_{K}^{*} \approx 150$. By multiplying this ratio by the observed stellar $K$-band luminosity of $L_{K}^{*}=1.5 \times 10^{6} L_{\odot}$, we find that the star formation in this region has $L_{\text {bol }}^{*}=2.3 \times 10^{8} L_{\odot}$ and accounts for $1.4 \%$ of the bolometric luminosity of the entire galaxy $\left(L_{\mathrm{bol}}=1.7 \times 10^{10} L_{\odot}\right.$, Maiolino et al. 1998).

Let us now assume a screen extinction of $A_{V}=9 \mathrm{mag}$, as suggested by Maiolino et al. (1998) for the nuclear region. Since $A_{K} \approx A_{V} / 10$ (Howarth 1983), $L_{K}^{*}$ is $\sim 2$ times that observed. This consideration has no effect on the $E W(\mathrm{Br} \gamma)$, but it reduces the $v_{\mathrm{SN}} / L_{K}^{*}$ by a factor of two. This new constraint does not change the prefered star formation scenario, still suggesting that the star formation is very young. The bolometric luminosity attributable to the nuclear star formation in this case would be $L_{\mathrm{bol}}^{*}=4.5 \times$ $10^{8} L_{\odot}$.

A perhaps more physical model than screen extinction is mixed extinction, where the dust and gas are uniformly mixed with the stars. In either case, the near infrared color excess is defined as

$E_{H-K}=-2.5 \log \left[\frac{I_{H} / I_{0 H}}{I_{K} / I_{0 K}}\right]$

where, $I_{H}$ and $I_{K}$ are the observed intensities in the two bands, and $I_{0 H}$ and $I_{0 K}$ are the intrinsic intensities. One can then use either $I_{\lambda} / I_{0 \lambda}=\mathrm{e}^{-\tau_{\lambda}}$ for the screen model or $I_{\lambda} / I_{0 \lambda}=\left(1-\mathrm{e}^{-\tau_{\lambda}}\right) / \tau_{\lambda}$ for the mixed model to derive the optical depth. With the usual wavelength scaling that $\tau_{V}=5.5 \tau_{H}=9.7 \tau_{K}$ one can then estimate the optical depth in the visual. The near infrared excess $E_{H-K}=0.45-0.60$ reported by Maiolino et al. (1998) then implies an extinction, after accounting for the galactic foreground, of $\tau_{V}=4.4-6.2$ for the screen model, and $\tau_{V}=12-25$ for the mixed model, similar to the estimate of Prieto et al. (2004). We note also that for the mixed model, the colour excess will saturate because if $\tau_{\lambda}$ is greater than a few, $I_{\lambda} / I_{0 \lambda} \sim 1 / \tau_{\lambda}$ so that the excess becomes $E_{H-K} \sim-2.5 \log \tau_{K} / \tau_{H}$. The limiting value of $E_{H-K}=0.6$ is consistent with the upper end of the measured range, suggesting that greater extinctions cannot be ruled out. We can, nevertheless, impose a very strong limit on the maximum possible extinction: the intrinsic luminosity from this nuclear star formation cannot exceed the galaxy's bolometric luminosity $\left(L_{\mathrm{bol}}^{*, \text { nuc }}<L_{\mathrm{bol}}\right)$. The lower limit, that $L_{\mathrm{bol}}^{* \text {,nuc }}>L_{\mathrm{bol}} / 75$, comes from the starburst model under the assumption of no extinction. The actual value of $L_{\mathrm{bol}}^{* \text { nuc }}$ is governed by the scaling of the starburst model. And this scaling depends directly on the ratio between the intrinsic and observed fluxes in the waveband where it is determined. Since the ratio between the intrinsic $K$-band and bolometric luminosities of the nuclear stellar component is fixed by the starburst model, it follows that the intrinsic $K$-band stellar luminosity must be less than 75 times the observed $K$-band stellar luminosity. Assuming a mixed model, one has $I_{K} / I_{0 K} \sim 1 / \tau_{K}$, implying that $\tau_{K} \sim 75$, equivalent to $\tau_{V} \sim 750$. A more meaningful, although less robust, limit is found if one excludes the circumnuclear star formation which Maiolino et al. (1998) estimated to account for $\sim 10^{10} L_{\odot}$. In this 

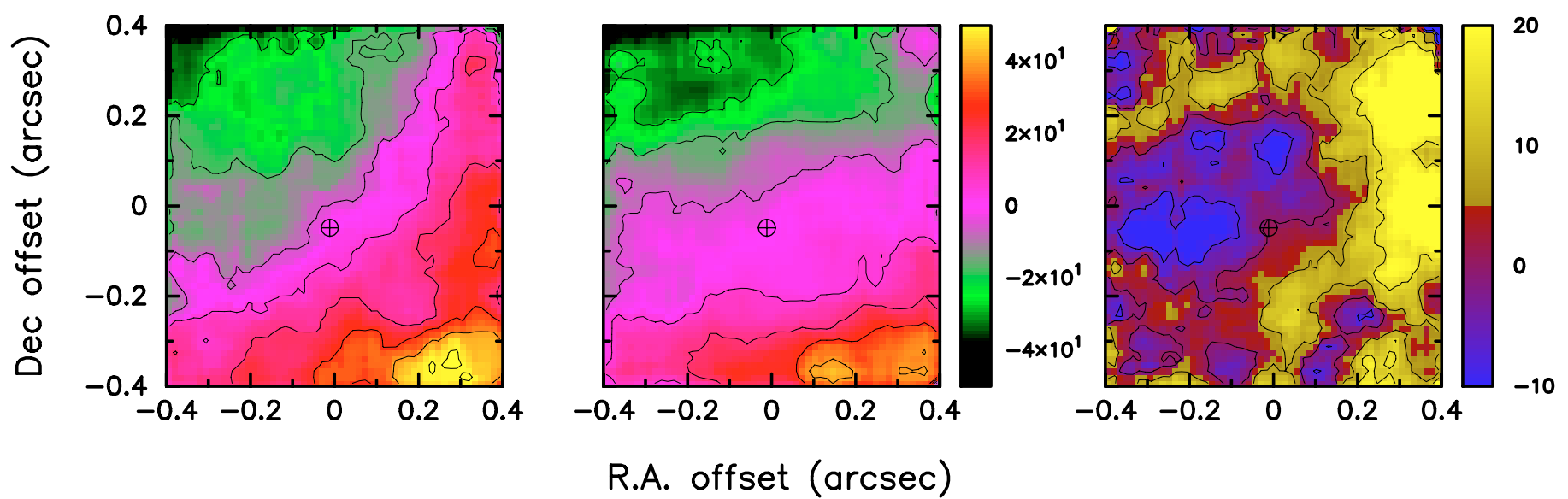

R.A. offset $(\operatorname{arcsec})$

Fig. 7. Velocity maps extracted from the SINFONI data cube in the central $0.8^{\prime \prime} \times 0.8^{\prime \prime}$ of Circinus. The maps show, from left to right: $\mathrm{H}_{2} 1-0 \mathrm{~S}(1)$, $\mathrm{Br} \gamma$ and difference in the velocity fields of the two lines For the case of $\mathrm{H}_{2} 1-0 S(1)$ and $\mathrm{Br} \gamma$ velocity maps, the colour scale extends from $[-50,50] \mathrm{km} \mathrm{s}^{-1}$, and contours are spaced equally every $10 \mathrm{~km} \mathrm{~s}^{-1}$. For the difference in the velocity fields, the colour scale extends from $[-10,20] \mathrm{km} \mathrm{s}^{-1}$, and contours are spaced equally every $5 \mathrm{~km} \mathrm{~s}^{-1}$ The difference in the velocity maps ranges between $[-10,10] \mathrm{km} \mathrm{s}{ }^{-1}$, except for the western edge of the map, where a difference of $\sim 25 \mathrm{~km} \mathrm{~s}^{-1}$ is observed. An encircled cross indicates in each case the peak of the continuum emission.

case $L_{\text {bol }} / L_{\text {bol }}^{* \text { nuc }}<30$ and the maximum optical depth would be of order $\tau_{V} \sim 300$. Although these limits are large, they are important and further discussion on the extinction will be presented in Sect. 7.

\section{Gas kinematics and mass in the nuclear region}

In Fig. 7 the projected velocity maps of the $\mathrm{H}_{2}$ and the $\mathrm{Br} \gamma$ lines are presented, as well as the difference in the velocity fields of the two lines. The maps for both emission lines are broadly consistent, exhibiting a gradual increase in velocity from north to south along a position angle (PA) of $\sim 18^{\circ}$ for the $\operatorname{Br} \gamma$ and $\sim 25^{\circ}$ (excluding the righthand side, see below) for the $1-0 S(1)$. Given the typical uncertainty of $5-10^{\circ}$ in each of these, they are consistent. They are the same as the galaxy's major axis (Freeman et al. 1977), a strong indication of pure galaxy rotation, and suggesting that there are no warps in the galaxy down to scales at least as small as a few parsecs. At smaller scales (from 0.1 to $\sim 0.4 \mathrm{pc}$ ), the maser emission traces a warped, edgeon accretion disk, which changes the PA from $\sim 29^{\circ}$ at $0.1 \mathrm{pc}$ to $\sim 56^{\circ}$ at $0.4 \mathrm{pc}$ (Greenhill et al. 2003). Our results suggest that at larger scales $(\sim 1 \mathrm{pc})$, possibly at the starting point of the torus, the galaxy's position angle has reverted back to $\sim 25^{\circ}$.

The fact that the PA for the $\mathrm{H}_{2} 1-0 S$ (1) velocity field appears to be greater is due primarily to the change in velocity gradient in a $0.3^{\prime \prime}$ strip along the western edge of the map; excluding this region, the difference between the maps is statistically insignificant (see Fig. 7 right image). The western region coincides with the extension of emission in the $\mathrm{H}_{2} 1-0 S$ (1) flux map and seems likely to be associated with a molecular outflow in (or at the edge of) the ionisation cone, perhaps radiatively driven by the AGN. It is important to notice that the observed velocity field of the $\mathrm{Br} \gamma$ line is clearly different to the one expected if it was associated with the NLR of the AGN, which would exhibit an increase in velocity in the western direction along the minor axis of the galaxy, as an outflow in the ionisation cone.

By correcting the velocity maps for inclination $\left(i=65^{\circ}\right)$, we find a rotation velocity of $75 \mathrm{~km} \mathrm{~s}^{-1}$ at $8 \mathrm{pc}$ from the nucleus. The velocity dispersion of the stars in the nuclear region is almost constant with a value of $\sigma_{\text {obs }} \approx 80 \mathrm{~km} \mathrm{~s}^{-1}$. By correcting the measured dispersion for the instrumental resolution corresponding to $\sigma_{\text {inst }} \approx 32 \mathrm{~km} \mathrm{~s}^{-1}$, we obtain an intrinsic dispersion of $\sigma_{z} \approx 70 \mathrm{~km} \mathrm{~s}^{-1}$, yielding a velocity to dispersion ratio of $V_{\text {rot }} / \sigma_{z} \approx 1.1$. This borderline ratio suggests that while there is significant rotation, random motions are also important - and on these scales perhaps dominant. Indeed, the kinetic energy in random motions is of order $3 \sigma^{2}$ while that in the ordered rotation is $V_{\text {rot }}^{2}$. This would imply that the distribution of gas is rather thick - consistent with our suggestion in Sect. 5 that the symmetry of the observed isophotes is due to the physical height of the nuclear region.

Further evidence that this is the case comes from the scale height derived if one assumes that the gas and stars are self gravitating, although this should only really be applied to a thin disk. In this case

$\sigma_{z}^{2}=2 \pi \mathrm{G} \Sigma z_{0}$

where $\sigma_{z}$ is the velocity dispersion of the stars, $\Sigma$ is the surface density of the disk, and $z_{0}$ is a characteristic measure of the scale-height of the disk perpendicular to the plane. In the nuclear region at $R<0.4^{\prime \prime}(8 \mathrm{pc})$, we estimate the mass of the gas and the stars residing in the disk to be $1.7 \times 10^{7} M_{\odot}$ (see also Sect. 7), yielding a mean surface density of $\Sigma=8.5 \times 10^{4} M_{\odot} \mathrm{pc}^{-2}$. By considering an intrinsic $\sigma_{z}=70 \mathrm{~km} \mathrm{~s}^{-1}$, we obtain a height of $z_{0}=2 \mathrm{pc}$, consistent with our earlier estimate based on the isophotal symmetry.

We suggest that this thickness does not necessarily imply that there is a distinct spheroidal structure in the central few parsecs; but that we are only looking at the very inner region of a larger disk which extends out perhaps as far as circumnuclear ring. By measuring the rotation velocity within a truncated field of view i.e. before $V_{\text {rot }}$ reaches its asymptotic value - one would underestimate $V_{\text {rot }} / \sigma$. It is also possible that either dynamics associated with the black hole, or heating from the AGN or local intense star formation, have caused the inner region of the disk to thicken.

For systems where the velocity dispersion is significant, estimating the mass from the rotational velocity alone leads to an underestimate. Accounting for random motions is not trivial, but can be approximated by $M_{\mathrm{dyn}}=\left(V_{\mathrm{rot}}^{2}+3 \sigma^{2}\right) r / G$. Hence we obtain a dynamical mass for the nucleus within $\pm 0.4^{\prime \prime}$ of $M_{\text {dyn }}=3.5 \times 10^{7} M_{\odot}$. 
Table 2. Properties of the sample of luminous and ultraluminous infrared galaxies used to estimate $L_{(1-0) S(1)} / M_{\text {gas }}$.

\begin{tabular}{|c|c|c|c|c|c|c|}
\hline $\begin{array}{l}\text { Galaxy } \\
\text { (1) }\end{array}$ & $\begin{array}{c}\log {\frac{L_{(1-0) S(1)}}{L_{\odot}}}^{a} \\
\text { (2) }\end{array}$ & $\begin{array}{c}\log \frac{M_{\text {gas }}}{M_{\odot}} \\
\text { (3) }\end{array}$ & $\begin{array}{c}\log \frac{L_{\mathrm{IR}}}{L_{\odot}} \\
\text { (4) }\end{array}$ & $\begin{array}{c}\log \frac{L_{(1-0) S(1)}}{L_{\mathrm{IR}}} \\
(5)\end{array}$ & $\begin{array}{c}\log \frac{L_{(1-0) S(1)}}{M_{\mathrm{gas}}} \\
(6)\end{array}$ & $\begin{array}{c}\text { References }^{b} \\
\text { (7) }\end{array}$ \\
\hline IR 01364-1042 & 6.7 & 10.2 & 11.8 & -5.0 & -3.4 & 1 \\
\hline IR 05189-2524 & 7.0 & 10.4 & 12.1 & -5.1 & -3.4 & 1 \\
\hline IR 09111-1007 & 6.9 & 10.4 & 11.9 & -5.0 & -3.4 & 1 \\
\hline IR $14378-3651$ & 6.8 & 10.2 & 12.1 & -5.3 & -3.3 & 1 \\
\hline IR 17208-0014 & 7.3 & 10.6 & 12.3 & -5.1 & -3.4 & 1 \\
\hline NGC 1614 & 6.2 & 9.7 & 11.6 & -5.3 & -3.5 & 2 \\
\hline NGC 2623 & 6.5 & 10.0 & 11.5 & -5.0 & -3.5 & 2 \\
\hline IR $10173-0828$ & 5.8 & 9.9 & 11.8 & -5.9 & -4.1 & 2 \\
\hline NGC 6090 & 6.7 & 10.5 & 11.5 & -5.0 & -4.0 & 2 \\
\hline NGC 6240 & 8.0 & 10.6 & 11.8 & -3.9 & -2.6 & 2 \\
\hline NGC 7469 & 6.4 & 10.2 & 11.6 & -5.2 & -3.8 & 2 \\
\hline Mrk 231 & 7.2 & 10.3 & 12.5 & -5.3 & -3.1 & 2 \\
\hline Mrk 273 & 7.3 & 10.6 & 12.1 & -4.8 & -3.3 & 2 \\
\hline Arp 220 & 7.0 & 10.5 & 12.2 & -5.2 & -3.5 & 2 \\
\hline NGC 695 & 6.4 & 10.6 & 11.7 & -5.2 & -4.2 & 3 \\
\hline NGC 1068 & 6.0 & 10.0 & 11.5 & -5.5 & -4.0 & 3 \\
\hline NGC 5135 & 6.2 & 10.2 & 11.1 & -4.9 & -4.0 & 3 \\
\hline
\end{tabular}

${ }^{a}$ Reference for $L_{(1-0) S(1)}$ data: Goldader et al. (1997), except for NGC 6090 Sugai et al. (2000).

${ }^{b}$ References for $M_{\text {gas }}$ data: (1) Mirabel et al. (1990); (2) Bryant \& Scoville (1999); (3) Gao \& Solomon (2004).

\section{Dimensioning the clumpy star forming torus in Circinus}

The spatial scales on which we have traced the gas and stars are similar to those associated with standard torus models (e.g. Pier \& Krolik 1992, 1993; Nenkova et al. 2002; Schartmann et al. 2005; Fritz et al. 2006). Indeed, the latter two authors have modelled Circinus specifically and find size scale for the torus of $30 \mathrm{pc}$ and $12 \mathrm{pc}$ respectively. However, to understand better the relation between the gas and the stars, we need, in addition to the stellar mass found in Sect. 5, to estimate the gas mass. Fortunately, as we discuss below, there is a way to do this from our data.

Table 2 lists 17 galaxies for which there are in the literature both measurements of the $\mathrm{H}_{2} 1-0 S$ (1) line flux and estimates of the total gas mass from mm CO 1-0 or CO 2-1 luminosities. The $\mathrm{H}_{2}$ 1-0 $S$ (1) luminosities are primarily from Goldader et al. (1997), since these are taken in large ( $3^{\prime \prime}$ or more) apertures and therefore are more likely to include all, or nearly all, the $\mathrm{H}_{2} 1-0 S$ (1) flux. In addition, these galaxies are actively star forming - as evidenced by their classification as luminous or ultraluminous galaxies. As the table shows, nearly all of these have similar ratios of the $1-0 S(1)$ luminosity with the infrared luminosity $L_{\mathrm{IR}}$, and also with the molecular mass estimated from the CO luminosity $L_{\mathrm{CO}}$. The former relation has previously been noted by Goldader et al. (1997), who suggested it could be explained in terms of supernova remnants if the $\mathrm{H}_{2}$ originates in gas shocked by the expanding shells. On the other hand, Davies et al. (2003) argued that in general the $\mathrm{H}_{2} 1-0 S$ (1) line in such galaxies is excited by fluorescence by young stars of gas dense enough that the lower vibrational levels are thermalised. In either case, if the $\mathrm{H}_{2}$ emission is primarily associated with star formation, it would not be unreasonable to find a correlation with $L_{\mathrm{IR}}$.

Similarly, a correlation between $L_{\mathrm{IR}}$ and $L_{\mathrm{CO}}$ has been known for a long time (see Young \& Scoville 1991 for a review). Recent work (Gao \& Solomon 2004) shows that while $L_{\mathrm{IR}}$ and $L_{\mathrm{CO}}$ are correlated, the logarithmic relation is linear only over limited luminosity ranges and there is a clear trend of $L_{\mathrm{IR}} / L_{\mathrm{CO}}$ (i.e. star formation efficiency) with luminosity. They also showed that if one uses instead a tracer of dense gas such as $\mathrm{HCN}$, the equivalent ratio $L_{\mathrm{IR}} / L_{\mathrm{HCN}}$ is constant; although for our application one then has the difficulty of estimating the gas mass from the $L_{\mathrm{HCN}}$.

In both relations, NGC 6240 is a major exception, having by far the largest $\mathrm{H}_{2} 1-0 \mathrm{~S}$ (1) luminosity. The reason is most probably that the excitation mechanism is most probably shocks rather than fluorescence (e.g. Sugai et al. 1997). For this reason, we have excluded NGC 6240 from the sample.

Given the correlations above, it would not be unreasonable also to expect a relation between 1-0 S(1) luminosity and $\mathrm{H}_{2}$ mass as traced by the CO luminosity (or even better would be $\mathrm{HCN}$ luminosity). In fact we find that the logarithmic ratio of these two quantities is $\log \frac{L_{(1-0) S(1)}}{M_{\text {gas }}}=-3.6 \pm 0.32$ which corresponds to $\sim 2.5 \times 10^{-4} L_{\odot} / M_{\odot}$ with a $1 \sigma$ uncertainty of a factor 2 . This is a quite remarkable result for such a diverse sample - that includes galaxies with powerful AGN for which X-ray irradiation of gas may be an important contributor to the 1-0 S(1) luminosity. It gives us confidence that we can use the $\mathrm{H}_{2} 1-0 S$ (1) line, without needing to know the details of how it is excited, to make at least an approximate estimate of the total (cold) molecular gas mass.

We apply this result to the nuclear region of Circinus because it has had recent vigorous star formation close to the AGN, and thus matches the sample. From the total $\mathrm{H}_{2} 1-0 S$ (1) luminosity of $4.4 \times 10^{3} L_{\odot}$ we estimate the total molecular mass to be $1.7 \times$ $10^{7} M_{\odot}$ - fully consistent with the dynamical mass estimated in Sect. 6. The gas mass surface density is then $8 \times 10^{4} M_{\odot} \mathrm{pc}^{-2}$, yielding a column density of $n_{H}=5.2 \times 10^{24} \mathrm{~cm}^{-2}$, which is very similar to that implied by X-ray observations (e.g. Matt et al. 1999). Since $n_{H} \approx 1.5 \times 10^{21} \tau_{V}$, the mean gas density implies an optical depth through the thickness of the disk of $\tau_{V}=3000$. This is far larger than the most likely values estimated in Sect. 5, and also inconsistent with the maximum possible optical depth.

This discrepancy suggests that the canonical torus in the unified model of AGN is not only forming stars but is a clumpy medium - so that much of the star light is not obscured despite the presence of huge gas column densities. 

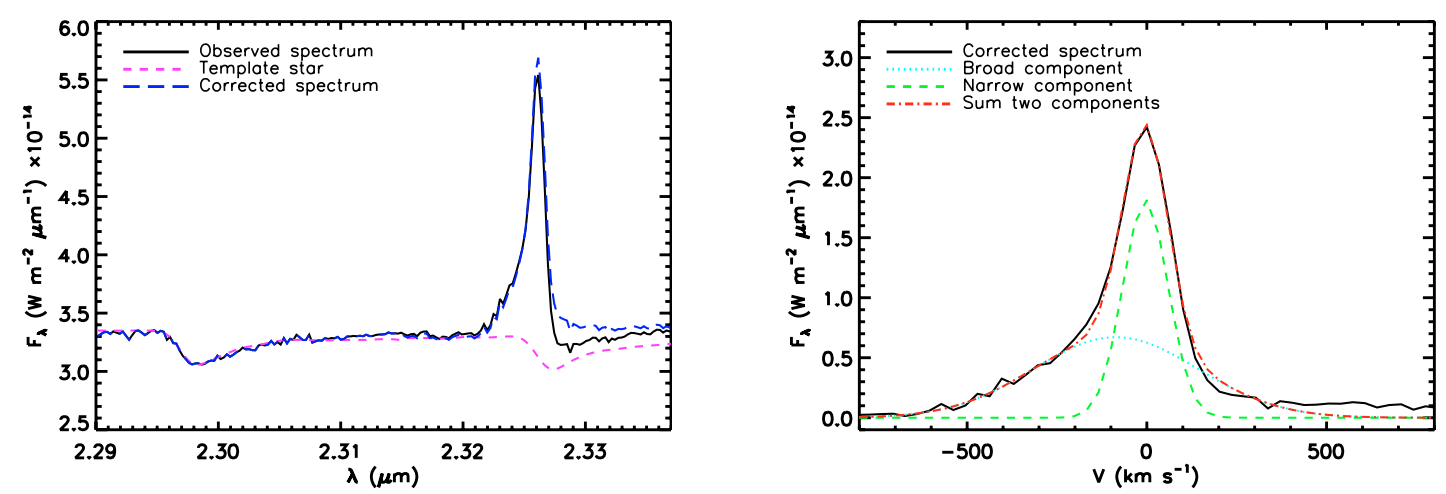

Fig. 8. Left panel: nuclear spectrum around [CaVIII] to show how the stellar continuum was substracted. Right panel: [CaVIII] profile in velocity space, fitted by two Gaussians.

\section{Coronal lines}

Because of the high ionization potential (IP $>100 \mathrm{eV}$ ) associated with Coronal Line Region (CLR) emission lines, highly energetic processes are required. The lines can be excited either by a hard UV to soft X-ray continuum, very hot collisionally ionized plasma, or a combination of both. If the excitation occurs via collisions, the gas temperature should be about $T=10^{6} \mathrm{~K}$. In the case of photoionization via the hard AGN continuum, temperatures of only a few $10^{3}-10^{4} \mathrm{~K}$ are needed.

In our observations of Circinus the [SivI], and [CaVIII] lines are extremely strong and [AlIX] is also detected, as apparent in the spectrum in Fig. 1. Due to the low signal to noise ratio of the $K$-band spectrum after $2.42 \mu \mathrm{m}$, we exclude the [SiVII] line at $2.48 \mu \mathrm{m}$ studied by Prieto et al. (2005). From this spectrum and also that in Fig. 8, it can be seen that the [CaVIII] line sits on top of the stellar ${ }^{12} \mathrm{CO}(3-1)$ absorption bandhead. A template star (HD 179323, shifted to the redshift of Circinus) was used to correct the stellar features in the spectrum and hence reconstruct the full [CaVIII] line profile. As the deep CO bandheads are produced in the atmospheres of red (super)giant stars which dominate the emission around $2.3 \mu \mathrm{m}$, HD 179323 (spectral type K0Iab) was choosen for this purpose. The correction was achieved by convolving the template with a Gaussian broadening function, and varying its parameters to minimise $\chi^{2}$, which was measured across the ${ }^{12} \mathrm{CO}(2-0)$ bandhead. The ${ }^{12} \mathrm{CO}(3-1)$ bandhead of the template was then convolved with the broadening function and subtracted from the galaxy's spectrum.

Figure 9 shows that in all three coronal line profiles we observe asymmetric and broadened lines, indicating the presence of two or more components within the coronal line region. In order to quantify the profile, we have fitted a superposition of two Gaussians to each line, as is summarised in Table 3. As can be seen in Fig. 8 for the case of [CaVIII], there is a strong narrow component and a weaker blueshifted broad component. These also have different spatial extents. Figure 10 shows azimuthally averaged radial profiles of the broad and narrow components of this line, as well as the PSF. It is clear that the broad component is resolved and spatially more extended. The flux peaks at about $1.5 \mathrm{pc}$ from the nucleus. On the other hand, the narrow component is unresolved and most of its flux comes from a region of less than $1 \mathrm{pc}$ across and coincident with the non-stellar $K$-band continuum peak (rather than the peak in $\mathrm{Br} \gamma, \mathrm{H}_{2} 1-0 S(1)$, and stellar continuum). These characteristics suggest that they originate in different regions around the AGN, and perhaps are even excited by different mechanisms.

In the case of the narrow component, the fact that it is compact and centered on the nucleus suggests that it originates

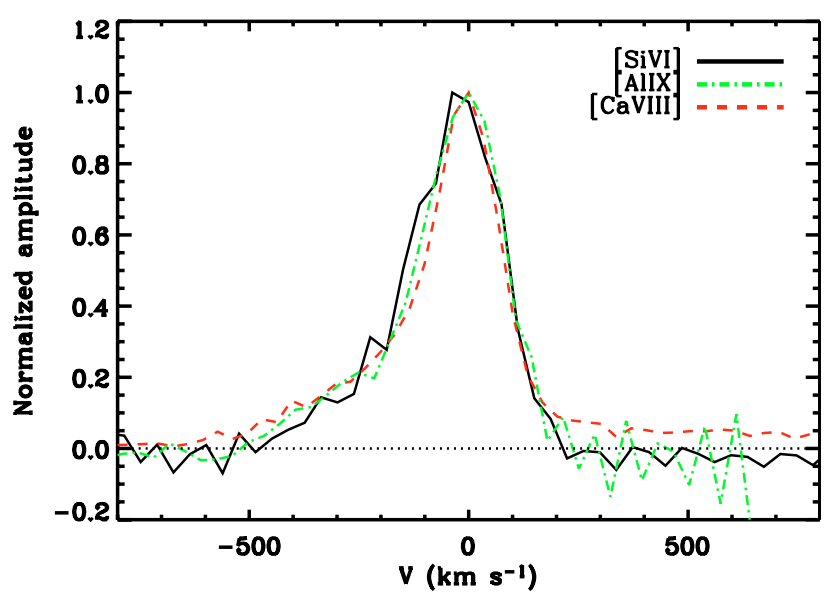

Fig. 9. Comparison of coronal line profiles in velocity space.

physically close to the AGN. Since the lines are narrow and at systemic velocity, these clouds of ionized material are not outflowing which is also an indication that they are excited by photoionization (Oliva et al. 1994, 1999).

In the case of the broad component, the fact that it is blue shifted indicates that part of the gas must arise in outflows around the AGN, as has been suggested by Rodríguez-Ardilla et al. (2004). Previous work on the coronal line emission in Circinus (Oliva et al. 1994) had ascribed the excitation to photoionisation. This was in part due to the narrow width of the most prominent component (typically $175 \mathrm{~km} \mathrm{~s}^{-1}$, from Table 3 ). However, the considerable broadening associated with the blue wing $\left(F W H M>300 \mathrm{~km} \mathrm{~s}^{-1}\right)$ opens the possibility that some fraction of it might in fact be excited by fast shocks. The picture of a shock-excited blue component is supported by the results of Prieto et al. (2005), who suggested that in addition to photoionization, shocks must contribute to the coronal emission. They proposed shock excitation as an additional energy source to explain the extended coronal line emission, as traced by [SivII], in a sample of 4 nearby Seyfert 2 galaxies. Although it could be a single broad component, we consider a more likely scenario to involve many narrow components arising from different clouds moving at different velocities whose emission combines to produce the observed morphology and profile. A mechanism by which broad coronal lines are created in out-flowing cloudlets which have been eroded from the main clouds is an intepretation which has been proposed for the detailed morphological and kinematic data available for NGC 1068 (Cecil et al. 2002). 
Table 3. Properties of the broad and narrow components of the detected coronal lines.

\begin{tabular}{|c|c|c|c|c|c|c|}
\hline Line & $\begin{array}{l}\text { IP } \\
(\mathrm{eV}) \\
(2)\end{array}$ & Component & $\begin{array}{c}\text { Flux } \\
\left(\mathrm{W} \mathrm{m}^{-2} \times 10^{-18}\right) \\
(4)\end{array}$ & $\begin{array}{c}F_{\text {broad }} / F_{\text {narrow }}{ }^{a} \\
\text { (5) }\end{array}$ & $\begin{array}{c}\text { Velocity } \\
\left(\mathrm{km} \mathrm{s}^{-1}\right) \\
(6)\end{array}$ & $\begin{array}{c}\text { FWHM Line Width } \\
\left(\mathrm{km} \mathrm{s}^{-1}\right) \\
(7)\end{array}$ \\
\hline [SiVI] & 167 & Broad & 26.8 & & -103 & 300 \\
\hline [SiVI] & 167 & Narrow & 24.7 & 1.1 & $\sim 0$ & 175 \\
\hline [AlIX] & 285 & Broad & 2.6 & & -228 & 300 \\
\hline [AlIX] & 285 & Narrow & 8.2 & 0.3 & $\sim 0$ & 200 \\
\hline [CavIII] & 127 & Broad & 28.0 & & -87 & 540 \\
\hline [CavIII] & 127 & Narrow & 20.0 & 1.4 & $\sim 0$ & 150 \\
\hline
\end{tabular}

${ }^{a}$ The ratio for each line is written in the row of the narrow component.

${ }^{b}$ Velocity shift from the systemic velocity.

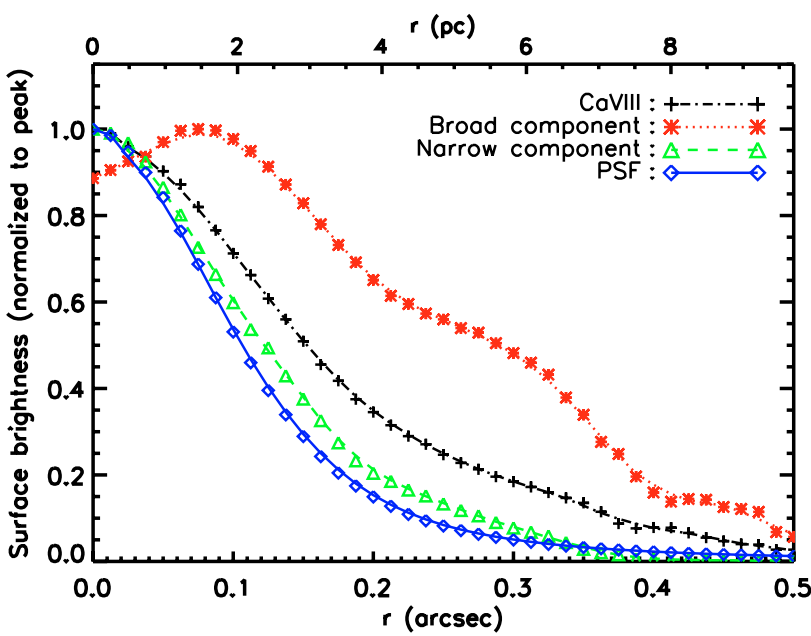

Fig. 10. Radial profile of the surface brightness of the [CaVIII] broad component (asterisks connected by a dotted line), and [CaVIII] narrow component (open triangles connected by a dashed line), compared to the [CaVIII] profile (crosses connected by a dotted-dashed line) and the PSF profile (open diamonds connected by a solid line).

It is interesting that we observe a correlation between the IP and the blueshift, as well as the $F_{\text {broad }} / F_{\text {narrow }}$, of the forbidden lines. As the ionization potential increases, the blueshift also increases and the $F_{\text {broad }} / F_{\text {narrow }}$ decreases. This can be understood if the acceleration of cloudlets away from the AGN is driven impulsively (which one might expect given the enormous variability of hard X-ray luminosity in many AGN) so that the cloudlets with the highest velocities will also be closest to the AGN - and as they travel further out, they will be decelerated by drag against the interstellar medium (probably small in the ionisation cone) and by the increasing gravitational pull. The gravitational pull is significant since it depends not only on the black hole mass, as assumed by Maiolino et al. (2000), but also on the gas and stars in the nuclear region which we have shown provide an order of magnitude more mass than the black hole alone on scales of $10 \mathrm{pc}$. Additionally, those lines which required the hardest ionising continuum will arise preferentially closer to the AGN. Thus although the strength of the narrow component which originates close to the AGN will depend on IP since there are fewer photons energetic enough to ionise species with higher IP; it will be even harder to create these lines in outflowing cloudlets, since they are already further from the AGN: they will arise only in the fastest clouds closest to the AGN. Lines with less extreme IP will be generated also in slower clouds further from the AGN and hence the mean blueshifted velocity will be less. Although we do not find a clear correlation between the IP and the width of the fit to the broad component, that of the [CaVIII] line exhibits the highest dispersion for the lowest IP, providing further support for this hypothesis that lines with the lower IP can arise in regions which are further from the AGN and hence moving outward slower.

\section{Conclusions}

We have presented near-infrared adaptive optics integral field spectroscopy of the nuclear region of the Circinus galaxy with an angular resolution of $0.2^{\prime \prime}$ and spectral resolution $R \sim 4200$, which we use to probe the gas and stellar morphologies and kinematics on scales of a few parsecs.

In the central $0.8^{\prime \prime}$, the non-stellar continuum dominates the $K$-band, contributing $85 \%$ of the total flux density. Offset by $\sim 0.15^{\prime \prime}$ from its peak are the $\mathrm{H}_{2} 1-0 S(1)$ and $\mathrm{Br} \gamma$ lines and stellar continuum. The similarity of their morphologies and kinematics lead us to conclude that they all originate predominantly with recent vigorous star formation. Analysis of these diagnostics together with the radio continuum suggest that within $8 \mathrm{pc}$ of the AGN there is a starburst which is exponentially decaying on a timescale of $100 \mathrm{Myr}$ and began only $80 \mathrm{Myr}$ ago, and currently has a bolometric luminosity of $\sim 1.4 \%$ that of the entire galaxy.

We have found that it is possible to make a reasonable estimate of the total gas mass from the $\mathrm{H}_{2} 1-0 S$ (1) line luminosity, finding that it is an order of magnitude more than the young stars, and also consistent with the column densities to the AGN implied by X-ray observations. Since the scales on which the gas and stars exist are similar, we suggest that the torus is forming stars; and because of the limits on extinction to the stars, that the torus must be a clumpy medium rather than uniform.

The coronal lines comprise two components: a prominent narrow $\left(F W H M \sim 175 \mathrm{~km} \mathrm{~s}^{-1}\right)$ part which is at systemic velocity, spatially unresolved (at our 4 pc $F W H M$ resolution), and centered on the non-stellar continuum; and a broad (FWHM > $300 \mathrm{~km} \mathrm{~s}^{-1}$ ) blue shifted part which is spatially extended. We argue that the narrow part arises in clouds physically close to the AGN; and that the blue wing originates, as appears to be the case in some other Seyfert galaxies, from cloudlets that have been eroded form the main clouds and are accelerated outward.

Acknowledgements. The authors are grateful to Almudena Prieto for kindly providing the $K$-band NACO image used for estimation of our spatial resolution. The authors are most gratefull to the entire SINFONI team from ESO, MPE, and NOVA for their support in the SINFONI comissioning.

\section{References}

Abuter, R., et al. 2005, SINFONI Data Reduction Software,to be published in the Proceedings of the Workshop IFS techniques and data reduction, 4-8 July, 
CfaI Durham, ed. J. Gerssen, J. Allington-Smith, \& D. Robertson, in a special edition of New Astronomy Reviews (Elsevier)

Bonnet, H., et al. 2003, Implementation of MACAO for SINFONI at the VLT, in NGS and LGS modes, in Adaptive Optical System Technologies II, ed. P. Wizinowich, \& D. Bonaccini, Proc. SPIE, 4839, 329

Bonnet, H., et al. 2004, First Light of SINFONI at the VLT, in The ESO Messenger, 117, 17

Bryant, P. M., \& Scoville, N. Z. 1999, ApJ, 117, 2632

Cecil, G., Dopita, M. A., Groves, B., et al. 2002, ApJ, 568, 627

Condon, J. J. 1992, ARA\&A, 30, 575

Davies, R. I., Forbes, D. A., Ryder, S., et al. 1998, MNRAS, 293, 189

Davies, R., Sternberg, A., Lehnert, M., \& Tacconi-Garman, L. 2003, ApJ, 597, 907

Davies, R., Tacconi, L., Genzel, R., Ott, T., \& Rabien, S. 2004, in Advancements in Adaptive Optics, ed. D. Bonnacini, B. Ellerbroek, \& R. Raggazoni, Proc. SPIE, 5490, 473

Davies, R. I., et al. 2006, in preparation

Eisenhauer, F., et al. 2003a, The Universe in 3D: First Observations with SPIFFI, the Infrared Integral Field Spectrometer for the VLT, in The ESO Messenger, 113, 17

Eisenhauer, F., et al. 2003b, SINFONI - Integral field spectroscopy at 50 milli-arcsecond resolution with the ESO VLT, in Instrument Design and Performance for Optical/Infrared Ground-based Telescopes, ed. M. Iye, \& A. Moorwood, Proc. SPIE, 4841, 1548

Freeman, K. C., Karlsson, B., Lyngå, G., et al. 1977, A\&A, 55, 445

Fritz, J., Franceschini, A., \& Hatziminiaoglou, E. 410 2006, MNRAS, 366, 767

Gao, Y., \& Solomon, P. M. 2004, ApJ, 606, 271

Goldader, J. D., Joseph, R. D., Doyon, R., \& Sanders, D. B. 1997, ApJS, 108, 449

Greenhill, L. J., Booth, R. S., Ellingsen, S. P., et al. 2003, ApJ, 590, 162

Howarth, I. D. 1983, MNRAS, 203, 301

Lawrence, A. 1987, PASP, 99, 309
Lucy, L. B. 1974, AJ, 79, 745

Maiolino, R., Krabbe, A., Thatte, N., \& Genzel, R. 1998, ApJ, 493, 650

Maiolino, R., Alonso Herrero, A., Anders, S., et al. 2000, ApJ, 531, 219

Marconi, A., Moorwood, A. F. M., Salvati, M., \& Oliva, E. 1994, A\&A, 291, 18

Marconi, A., Moorwood, A. F. M., Origlia, L., \& Oliva, E. 1994, The ESO Messenger, 78, 20

Matt, G., Fiore, F., Perola, G., et al. 1996, MNRAS, 281, L69

Matt, G., Guainazzi, M., Maiolino, R., et al. 1999, A\&A, 341, L39

Meurer, G. R., Heckman, T. M., Leitherer, C., et al. 1995, ApJ, 110, 2665

Mirabel, I. F., Booth, R. S., Garay, G., Johansson, L. E. B., \& Sanders, D. B. 1990, A\&A, 236, 327

Nenkova, M., Ivezíc, Ž., \& Elitzur, M. 2002, ApJ, 570, L9

Oliva, E., Salvati, M., Moorwood, A. F. M., \& Marconi, A. 1994, A\&A, 288, 457

Oliva, E., Marconi, A., Cimatti, A., di Serego \& Alighieri, S. 1998, A\&A, 329, L21

Oliva, E., Marconi, A., \& Moorwood, A. F. M. 1999, A\&A, 342, 87

Pier, E., \& Krolik, J. 1992, ApJ, 401, 99

Pier, E., \& Krolik, J. 1993, ApJ, 418, 673

Prieto, M. A., Meisenheimer, K., Marco, O., et al. 2004, ApJ, 614, 135

Prieto, M. A., Marco, O., \& Gallimore, J. 2005, MNRAS, accepted

Rodríguez-Ardilla, A., Pastoriza, M., Viegas, S., Sigut, T., \& Pradhan, A. 2004, A\&A, 425, 457

Schartmann, M., Meisenheimer, K., Camenzind, M., Wolf, S., \& Henning, Th. 2005, A\&A, 437, 861

Sternberg, A. 1998, ApJ, 506, 721

Sugai, H., Malkan, M., Ward, M., Davies, R., \& McLean, I. 1997, ApJ, 481, 186

Sugai, H., Davies, R., Ishii, M., \& Ward, M. 2000, MNRAS, 317, 447

Thatte, N., Quirrenbach, A., Genzel, R., Maiolino, R., \& Tecza, M. 1997, ApJ, 490, 238

Thornley, M., Förster Schreiber, N., Lutz, D., et al. 2000, ApJ, 539, 641

Young, J., \& Scoville, N. 1991, ARA\&A, 29, 581 